\title{
Bovine three-portion pericardial patch for reconstruction of the aorto-mitral curtain in infective endocarditis
}

\author{
Atsushi Hiromoto, Shun-Ichiro Sakamoto* (D, Yasuo Miyagi and Takashi Nitta
}

\begin{abstract}
Background: Surgery for infective endocarditis involving the aorto-mitral curtain (AMC) is challenging and requires extensive incisions and complex reconstruction procedures. However, in patients with preserved aortic annulus, reconstruction of the $A M C$ is possible using a simple technique with limited incisions.

Case presentation: A handmade bovine three-portion pericardial patch was used to reconstruct the AMC in a patient with severe endocarditis requiring double valve replacement; the technique allowed for steady anchorage of prosthetic valves without additional incisions other than conventional aortotomy and atriotomy. Postoperative echocardiography revealed normal cardiac function and no significant perivalvular leakage. The patient displayed complete recovery and was discharged on postoperative day 33. The patient was symptom-free at his 1-year follow-up and displayed normal laboratory and echocardiographic findings.
\end{abstract}

Conclusion: The bovine three-portion pericardial patch is useful for reconstructing the AMC in patients with infective endocarditis accompanied by preserved aortic annulus.

Keywords: Infective endocarditis, Aorto-mitral curtain, Patch repair

\section{Background}

Involvement of the aorto-mitral curtain (AMC) is uncommon in patients with infective endocarditis. Once it is affected, the procedure for double valve replacement becomes more complex as it necessitates reconstruction of the AMC. This surgery is challenging and is associated with a high mortality rate $(20-30 \%)$ [1-3]. In contrast, in patients with preserved aortic annulus, it is possible to reconstruct the AMC using a simple technique requiring limited incisions. Here, we report the surgical case of a patient with infective endocarditis with AMC involvement who was treated using the conventional approach.

\section{Case presentation}

A 57-year-old man was admitted to the hospital due to hyperleukocytosis. Echocardiography revealed irregularly shaped vegetation (size, $25 \times 15 \mathrm{~mm}$ ) attached to

\footnotetext{
* Correspondence: saka-165@nms.ac.jp

Department of Cardiovascular Surgery, Nippon Medical School, 1-1-5 Sendagi, Bunkyo-ku, Tokyo 113-8603, Japan
}

the anterior leaflet of the mitral valve. The vegetation exhibited oscillation and was connected to the thickened aortic valve. Color flow imaging showed severe insufficiency of both the aortic and mitral valves with perforation in the AMC (Fig. 1). Chest X-ray revealed bilateral lung congestion due to acute heart failure. Therefore, emergency surgery was indicated.

The heart was approached via median full sternotomy. An oblique incision was made in the ascending aorta under conditions of cardiac arrest. The aortic valve was bicuspid (type 1). Vegetation was observed at the non-coronary cusp, extending to the AMC. The mitral valve was exposed via the superior trans-septal approach. The anterior leaflet was thickened and had attached vegetation. Debridement of the infected tissue led to a defect in the middle portion of the anterior mitral annulus, AMC, and non-coronary cusp.

For reconstructing the defective parts, a glutaraldehyde-treated bovine pericardial patch (Model 4700, Edwards Lifesciences, Irvine, CA, USA) was folded to make a three-portion patch (Fig. 1a). The triangular portion (AMC portion) of two pericardial patches was sutured 


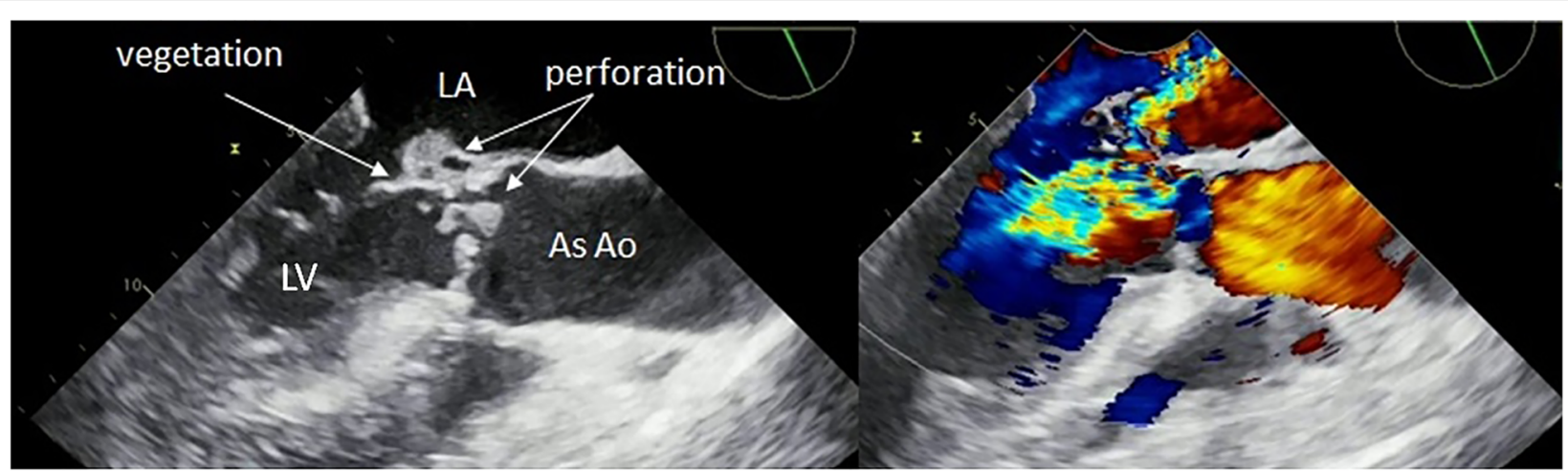

Fig. 1 Three-chamber transoesophageal echocardiographic view reveals vegetation (size, $25 \times 15 \mathrm{~mm}$ ) attached to the anterior leaflet of the mitral valve. Color flow imaging reveals severe insufficiency of both the aortic and mitral valves with perforation in the AMC and at the base of the non-coronary cusp. LV left ventricle, LA left atrium, AsAo ascending aorta, AMC aorto-mitral curtain

to the AMC remnant using continuous sutures. Pledgeted everted mattress sutures were placed around the mitral annulus, and the anterior rim was reconstructed with the pericardial patch (MV portion). A 23-mm mechanical valve (Abbott
Laboratories, Chicago, IL, USA) was tied down in the intra-annular position of the aortic annulus in a manner wherein the sutures pass through the aortic annulus and the rectangular portion (AV portion) of the pericardial patch. Finally, a $28-\mathrm{mm}$ mechanical

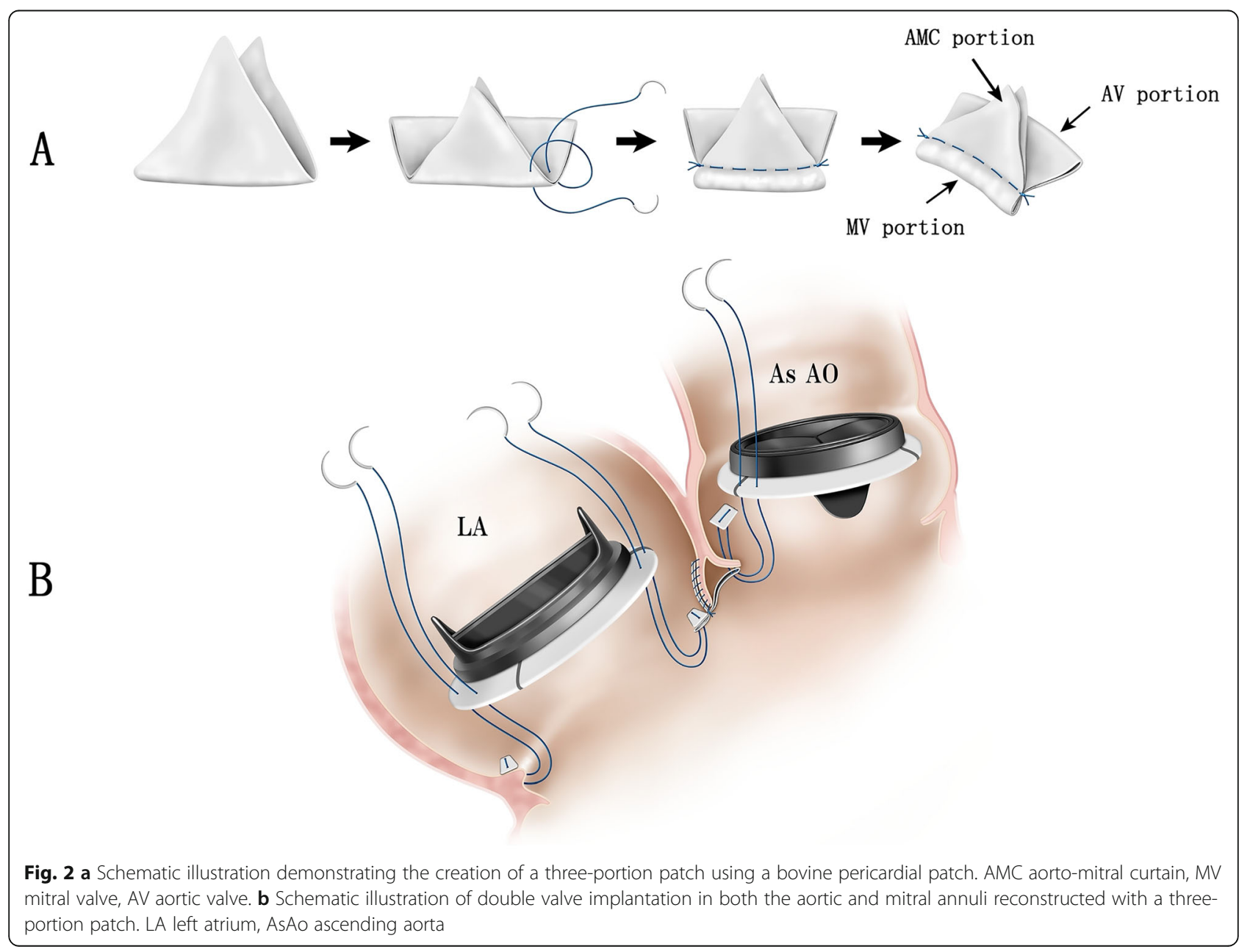


valve (Abbott Laboratories, Chicago, IL, USA) was tied down in the mitral annulus (Fig. 2b).

Cardiobacterium valvarum was isolated on blood culture. Vancomycin and ceftriaxone were intravenously administered for 4 weeks postoperatively. Postoperative echocardiography revealed normal cardiac function with no significant perivalvular leakage. The patient displayed complete recovery and was discharged on postoperative day 33. The patient was symptom-free at his 1-year follow-up and exhibited normal laboratory and echocardiographic findings.

\section{Discussion}

Several studies have reported on the most appropriate AMC reconstruction technique depending on the extent of involvement. For example, David et al. reported a technique for patch reconstruction of the anterior mitral annulus, left ventricular outflow tract, and left atrial roof in patients with an infected AMC [1]. Davierwala et al. used an aortic prosthesis or aortic root conduit in patients with more extensive aortic annular involvement [3]. Moreover, Tedoriya et al. reported a handmade aorto-mitral bioprosthetic valve created using two bioprosthetic valves and Dacron knitted fabric for reconstructing the AMC after extensive debridement [4]. These techniques require complex incisions in addition to routine aortotomy and atriotomy to expose the aortic and mitral valves.

In our case, aortotomy was performed to observe the aortic valve because the periannular tissue damage was not clearly identified via both preoperative and intraoperative echocardiography. In patients with involvement of the aortic annulus, annular incision using the Manouguian technique might be an appropriate add-on approach to mitral valve surgery [5]. However, this approach inevitably requires double valve replacement on the single layer of the pericardial patch. The advantage of our technique is that it allows reconstruction of the AMC without any additional incisions other than aortotomy and atriotomy; this facilitates the repair of the mitral valve or the implantation of two prosthetic valves in the natural annular position without excessive suture tension.

Use of the bovine pericardial patch is helpful in surgery for active infective endocarditis. Shinn et al. reported the use of a pericardial patch for reconstruction of the aortic annulus, AMC, and mitral annulus in 57 patients. They reported satisfactory outcomes at mid-term follow-up, thereby indicating the safety and durability of such patches [6]. However, complications associated with extensive use of the pericardium, such as valve dehiscence, perivalvular leakage, and pseudo-aneurysms, have been reported within the first year of surgery [3]. In our technique, the three-portion patch was created as a double layer. The shape of each patch portion was designed considering the suture line for the reconstruction of the AMC. The AMC patch portion was fitted in the triangular area surrounded by the left and right trigone and the mitral annulus. The aortic patch portion was rectangular and was horizontally sutured to the aortic annulus. We believe that this minimum usage of the double layer patch and the modified design for the AMC will render the mitral patch portion more durable and allow for anchorage of the prosthetic valve in patients requiring debridement of the AMC. In contrast, calcification of the pericardial patch has been reported particularly when used in the posterior rim of the mitral annuls. Dacron polyester fabric is an alternative material that may be preferable in this situation [7]. Further follow-up is required to ascertain the long-term durability of the pericardial patch.

\section{Conclusion}

The bovine three-portion pericardial patch is useful for the reconstruction of the AMC in patients with infective endocarditis with preserved aortic annulus.

\section{Abbreviations \\ AMC: Aorto-mitral curtain; AV: Aortic valve; MV: Mitral valve}

Acknowledgements

None

Funding

None

Availability of data and materials

Data sharing is not applicable to this article as no datasets were generated or analyzed during the current study.

Authors' contributions

$\mathrm{AH}$ wrote the initial draft of the manuscript. SS have contributed to revision of the manuscript and preparation for submitting. YM and TN have contributed to interpretation and critically reviewed the manuscript. All authors read and approved to submit the final manuscript.

Ethics approval and consent to participate

Not applicable

Consent for publication

Written informed consent was obtained from the patient for publication of this case report and accompanying images.

Competing interests

The authors declare that they have no competing interests.

\section{Publisher's Note}

Springer Nature remains neutral with regard to jurisdictional claims in published maps and institutional affiliations.

Received: 13 November 2018 Accepted: 18 December 2018 Published online: 04 January 2019

\section{References}

1. David TE, Kuo J, Armstrong S. Aortic and mitral valve replacement with reconstruction of the intervalvular fibrous body. J Thorac Cardiovasc Surg. 1997:114:766-72.

2. Sheikh AM, Elhenawy AM, Maganti M, Armstrong S, David TE, Feindel CM. Outcomes of double valve surgery for active infective endocarditis. J Thorac Cardiovasc Surg. 2009;138:69-75. 
3. Davierwala PM, Binner C, Subramanian S, Luehr M, Pfannmueller B, Etz C, Dohmen P, Misfeld M, Borger MA, Mohr FW. Double valve replacement and reconstruction of the intervalvular fibrous body in patients with active infective endocarditis. Eur J Cardiothorac Surg. 2014:45:146-52.

4. Tedoriya T, Hirota M, Ishikawa N, Omoto T. Reconstruction of aorto-mitral continuity with a handmade aorto-mitral bioprosthetic valve for extensive bivalvular endocarditis. Interact Cardiovasc Thorac Surg. 2013;16:405-7.

5. Nishimura T, Ota T, Takayama H, Naka Y. Extended trans-septal and left atrial dome incision combined with Manouguian's aortotomy for complex double valve surgery. Eur J Cardiothorac Surg. 2014;46:127-8.

6. Shinn SH, Sung K, Park PW, Lee YT, Kim WS, Jun TG, Lee SC, Park SW. Results of annular reconstruction with a pericardial patch in active infective endocarditis. J Heart Valve Dis. 2009;18:315-20.

7. De Oliveira NC, David TE, Armstrong S, Ivanov J. Aortic and mitral valve replacement with reconstruction of the intervalvular fibrous body: an analysis of clinical outcomes. J Thorac Cardiovasc Surg. 2005;129(2):286-90

\section{Submit your manuscript to a SpringerOpen ${ }^{\circ}$ journal and benefit from:}

- Convenient online submission

- Rigorous peer review

- Open access: articles freely available online

- High visibility within the field

- Retaining the copyright to your article

Submit your next manuscript at $\boldsymbol{\wedge}$ springeropen.com 